\title{
Some Probabilistic Aspects of Fire Risk Analysis for Nuclear Power Plants
}

\author{
GEORGE APOSTOLAKIS \\ School of Engineering and Applied Science \\ University of California \\ Los Angeles, California 90024, USA
}

ABSTRACT

The development of a methodology for the quantification of the risks associated with fires in nuclear power plants requires the use of judgment, deterministic models and statistical evidence. Several issues that arise are discussed including: 1. The impact of design changes, plant-to-plant variation and other qualitative factors on the assessment of the frequency of fires in specific locations; 2. The importance of distinguishing among statistical, parameter and modeling uncertainties, when fire propagation is studied; 3 . How parameter and modeling uncertainties can be assessed; and 4. How the probability distribution of detection and suppression times can be determined.

\section{INTRODUCTION}

The evaluation of the risk associated with fires in nuclear power plants has attracted considerable attention in the last few years. While it was believed earlier, on the basis of crude estimates, that this risk was very small and, therefore, dominated by other risks, more detailed analysis showed that the risks from fires could be significant. For example, fires constitute the second (after seismic) most important contribution to core melt frequency at Indian Point 2 (1). These fires (occurring at specific locations) could damage vital power cables, thus causing a small loss of coolant accident through the failure of the reactor coolant pump seals. At the same time, several other power cables could fail incapacitating vital safety components (safety injection pumps, containment spray pumps and fan coolers). The mean frequency of core melt due to fires was found to be $2.0 \times 10^{-4}$ per reactor year, while the 5 th and 95 th percentiles were $6.0 \times 10^{-6}$ and $7.6 \times 10^{-4}$ per reactor year.

The preceding example is fairly typical of the findings of a fire risk analysis for nuclear power plants and illustrates several of its salient features. The methodology is focused on the effects of fires on control and power cables and traces the impact of their failure on the plant. Furthermore, the scenarios that are identified have very low frequencies and special care must be taken to display the uncertainties. We have found that the Bayesian interpretation of probability (2-3) provides a convenient framework for the explicit and rigorous treatment of the uncertainties and the subjective judgments that are necessary.

The purpose of this paper is to discuss several of the statistical issues that arise when a fire risk analysis is carried out. Since the basic methodo- 
logy has been presented elsewhere (4-5), its details will. not be presented here. While this methodology has been developed for nuclear power plants, the issues that we discuss are relevant to any effort to quantify the risks associated with fires in any large and complex technological system. This belief is based on the realization that these issues are the consequences of two features that a11 such systems share: 1 . Fires must occur at the "right" location to serious$1 y$ threaten the safety of the system, and 2. The events of interest are rare, therefore, conventional statistical evidence is very weak and the analysts must devise methods that extract the maximum possible amount of information from this evidence.

\section{OVERVIEW OF THE METHODOLOGY}

The methodology is centered around the following three tasks:

1. The identification of the "critical" locations and the assessment of the frequency of fires.

2. The estimation of fire growth times and the competing detection and suppression times.

3. The response of the plant.

A location is declared as "critical", when the occurrence of a fire there has the potential of creating an abnormal condition in the plant and damaging the engineered safety functions (ESF). Thus, tasks 1 and 3 are related. As stated earlier, only fires that affect control and power cables are considered. Figure 1 shows a situation, where the cable trays are elevated. The plant analysis has already determined that trays $A, B$ and $C$ carry cables, which, if failed, would lead to undesirable consequences. The occurrence of a large fire on the floor, as shown, could fail these cables, therefore, this location merits a detailed analysis, and is "critical". In other situations the cable trays are closer to the floor and one worries about a fire starting on one tray and propagating to the others.
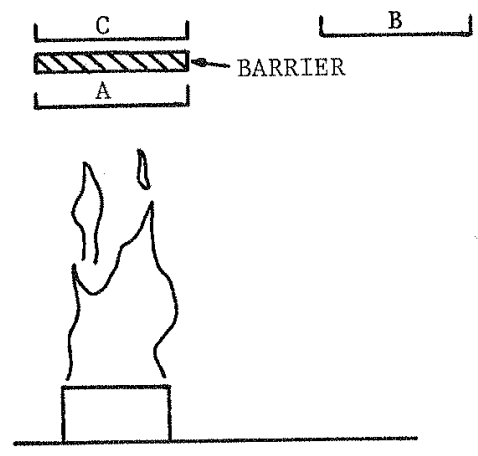

Figure 1. Sample Room Configuration.

In the second step the time $\mathrm{T}_{\mathrm{G}}$ that it takes for the fire to propagate and damage the cables of the trays is estimated using the computer code COMPBRN (6-7). This code uses empirical correlations for such quantities as the fire's mass burning rate and flame height to characterize the fire as a heat source. It also employs heat and mass balances to determine the extent and temperature of the layer of hot gases accumulating near the room ceiling. The heat transfered to objects within the room and the room boundaries is then calculated and the thermal response of these receivers determined. Damage is assumed to occur 
when the surface temperature of an object exceeds a predefined limit.

The detection and suppression times are representd by the "hazard" time $\mathrm{T}_{\mathrm{H}}$, i,e, the fire actually burns for a time $\mathrm{T}_{\mathrm{H}}(8)$. The potential occurrence of fire damage to components is modeled as the outcome of a competition between fire growth and fire suppression processess over time. The conditional frequencey of damage, given that the fire occurs, is

$\mathrm{Q}=\operatorname{Fr}\left\{\mathrm{T}_{\mathrm{G}}<\mathrm{T}_{\mathrm{H}} \mid\right.$ fire $\}$

where $\operatorname{Fr}\{\mathrm{A} / \mathrm{C}\}$, denotes the frequency of occurrence of event $\mathrm{A}$ conditioned on the occurrence of event C. Eq. (1) simply says that the frequency of damage, given a fire, equals the frequency by which the growth time is smaller than a hazard time, $i . e$, the time it takes to detect and suppress the fire.

The last step of the analysis (plant response) is highly plant specific and requires intimate knowledge of the plant. It essentially entails a detailed investigation (using, usually, fault and event trees) of whether the damaged cables can prevent the successful termination of the neutron chain reaction and whether the heat that is produced in the core after the shutdown (the "decay" heat) is successfully removed, thus avoiding a melt down. We note that the plant response is affected not only by the components damaged by the fire, but also by the components which are unavailable due to other causes (e.g., maintenance).

The analysis described above is carried out for all the critical locations. The total frequency of core melt due to fires is, then, the sum of the frequency contribution from each location, i.e.,

$\lambda_{\text {CM-Eire }}=\sum_{j} \lambda_{j} Q_{d}\left|j Q_{C M}\right| d, j$

where

$\lambda_{j}=$ frequency of fires of class $j$, where the classes are determined both by the location and initial severity of the fire (step 1)

$Q_{\mathrm{d} \mid j}=$ fraction of class $j$ fires which lead to damage to a specified set of components (step 2, see Eq. (1)), and

${ }^{Q} \mathrm{CM} \mid \mathrm{d}, j=$ fraction of class $j$ fires causing damage to the specified set of components which lead to core melt (step 3 ).

\section{THE FREQUENCY OF FIRES}

Modeling Needs

The first factor in eq. (2) to be estimated is the frequency of fires. The difficulties of doing so become evident, when we go back to Fig. 1 and realize the $\lambda_{f}$ represents fires that occur in the "right" location, i.e., beneath the cable trays or thereabout, and are of sufficient magnitude to cause damage to the cables. Each such situation is unique and it is impossible to find statistical evidence which is directly applicable. However, evidence regarding "similar" rooms or equipment in other plants is available ana must be utilized. Of course, what are similar rooms can not be detined precisely. the model which has been used considers $\lambda_{j}$ as the product of several factors, 
as follows:

$\lambda_{j}=\lambda_{C_{j}} \quad \mathrm{f}_{\mathrm{C}_{j}, \mathrm{~L}} \quad \mathrm{E}_{\mathrm{C}_{j}, L, S}$

where

$\lambda_{C_{j}} \quad=$ annual frequency of fires in class $C_{j}$ of rooms or equipment

$\mathrm{f}_{\mathrm{C}}, \mathrm{L}=$ fraction of these fires that occur in the appropriate 1ocation.

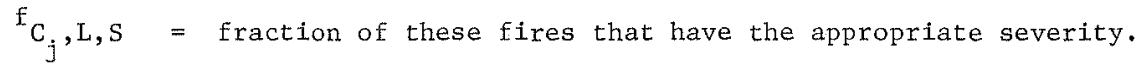

Each of these quantities is discussed below.

The Frequencies of Fires in Classes of Rooms or Equipment

The classes $C$, are obtained by dividing the plant into generic buildings, rooms, or equipment. Reference 9 presents one such division in which the frequencies of fires in auxiliary buildings, turbine buildings, control rooms, and cable spreading rooms are evaluated. It also presents the frequency of fires in reactor coolant pumps ( in a pressurized water reactor) per year of operation and the unavailability of a diesel generator unit due to fires. The general approach is to develop these distributions from Bayes' theorem (3), in which the prior distribution is noninformative and the likelihood function is determined by the statistical evidence. The latter creates problems of both interpretation and use.

Design and procedure changes may create questions about the applicability of some of the operating experience. For example, one plant's cable spreading rooms have experienced three relay fires within 5 months in the motor control center (MCC) cabinets that are installed there (9). These fires were due to a generic defect in certain relays. Corrective actions have since been taken in those units and in other plants that had the same kind of relays. Thus, we suspect that those fires were unique to that station at that time and their likelihood of recurrence has been substantially reduced. The question is, then, how many fires we should include in our data when we estimate the frequency of fires in these rooms. In this case the three incidents were counted as one, i.e., only the cause of these fires was counted. Of course, the design changes have, presumably, eliminated this cause, however, one can look at this situation from the point of view that there is a class of faflure causes that may occur and elimination of one specific cause does not eliminate the class as a whole.

In addition to the questions of interpretation of the evidence, another issue is whether all rooms or equipment in a class should be treated as being identical, when, in fact, there may be significant plant-to-plant variation. The plant-to-plant variability of the occurrence rates can be very important, especially when the event of interest has occurred in some plants but not in the majority of plants. Formal methods for developing generic distributions which explicitly model this variability have been developed (10). An example that shows how significantly different the results may be deals with the occurrence of fires in control rooms (9). The statistical evidence is of the form $\left\{k_{i}, T_{i}\right\}$, where $k_{1}$ is the number of fires over $T_{1}$ control room years at plant $i$. 
$\mathrm{T}_{i}$ is in the range of 1 to 20 years, while $k_{\text {, }}$ is zero for all but one 1 . Using formal methods we can derive a generic distribution for the frequency of fires which reflects the plant-to-plant variability and has characteristic values:

5th percentile: $1.3 \times 10^{-7}$ per control room year 50 th percentile: $3.2 \times 10^{-4}$ per control room year 95 th percentile: $1.5 \times 10^{-2}$ per control room year mean: $4.9 \times 10^{-3}$ per control room year

If, on the other hand we ignore the variablity of the rate of fires over plants and we say that the evidence is $k=\sum_{i} k_{i}=1$ fire over $\mathrm{T}=\sum_{i} \mathrm{~T}_{i}=453$ control room years, then the resulting characteristic values are:

5th percentile: $1.2 \times 10^{-4}$ per control room year 50th percentile: $1.5 \times 10^{-3}$ per control room year 95 th percentile: $6.6 \times 10^{-3}$ per control room year mean: $2.2 \times 10^{-3}$ per control room year

This distribution is much narrower than the first one. The plant-to-plant curve, being broader, has a higher 95 th percentile $\left(1.5 \times 10^{-2}\right)$ and a mean value which is more than twice that of the second distribution. While the first model is more realistic (in the sense that it allows for differences among plants), it is the second model that is used by most analysts, mainly because of lack of appreciation of the difference that the plant-tomplant variability can make.

Specialization Factors

The factors $f_{C_{j}, L}$ and $f_{C_{j}, L, S}$ of eq. (3) have to be assessed using judgment, since they are highly situation specific and statistical evidence does not exist. The ability of analysts to quantify their judgment in terms of probabilities has been studied extensively (for some observations pertinent to risk analysis see (3)). While it has been found that people, in general, are not very good at it, it has also been determined that the use of formal methods and training can improve this ability to a significant degree. Essentially, the assessors must try to develop numerical estimates, which fit "coherently" (11) in their body of knowledge and beliefs. Several considerations which generally help in the present context are:

1. The number of rooms that the buildings of class $C_{\text {; }}$ have. While all rooms are not necessarily equally likely to have the fire occur there, this number is a useful input.

2. The contents of the rooms. For example, rooms containing ofl or machinery with moving parts are more likely to have fires.

3. Even within a room a fire must occur in specific locations to cause damage, e.g., in Fig. 1, the fire must occur beneath the cable trays. This argument is, of course, intimately related to the types and quantities of fuel that could be reasonably assumed to be introduced into the room. This is a particularly thorny issue, since the amounts of oil, for instance, that have to be assumed present are not found, under normal conditions, in these rooms. On the other hand, there have been instances in which several gallons of oil have been left inadvertently in controlled areas. 
4. The frequency of visits by plant personnel.

Even though considerations of this kind do help the analysts, the fact remains that this is a "soft" and controversial part of the analysis.

\section{FIRE GROWTH}

As stated earlier, the computer code COMPBRN is used to calculate the time to damage a speciffed component, $\mathrm{T}_{\mathrm{G}}$. This time we call the deterministic reference model (DRM) estimate. The question, now, is what kinds of uncertainties are associated with this number.

The uncertainties associated with modeling of physical processes can be thought of as being of two kinds: the statistical uncertainty and the stateof-knowledge uncertainty. The statistical uncertainties stem from the random nature of fire; if we perform a particular experiment a large number of times under 'identical' conditions and measure $\mathrm{T}_{\mathrm{G}}$, we will obtain a frequency distribution. Our physical model cannot predict the environmental fluctuations which lead to this distribution. These uncertainties are inherent in the nature of fire. Even if our knowledge of fire were to increase dramatically, we would not be able to reduce these uncertainties significantly. Such an increase in knowledge, however, would markedly reduce our state-of-knowledge uncertainties.

There are two kinds of state-of-knowledge uncertainty: the parameter uncertainty and the modeling uncertainty. The parameter uncertainty is due to insufficient knowledge about what the input to the code should be. The mode1ing uncertainty is due to simplifying assumptions and the fact that the models used may not accurately model the true physical process.

The DRM is a collection of approximate models which are valid under certain conditions, The modeling uncertainty is due not only to our uncertainty in the accuracy of each model's predictions under the conditions they were developed for, but also to our uncertainty in the synthesis of these independent models. One of our primary concerns is whether or not the synthesis contains enough component models (i.e., if all important phenomena have been modeled).

The three types of uncertainty that we have discussed, i.e., statistical, parameter and modeling uncertainty, must be expressed in terms of probability distributions, which will be used in the risk assessment. The development of these distributions requires, again, substantial judgment, since conventional. statistical evidence is lacking. It is judged that the variation of the estimated propagation time is dominated by state-of-knowledge uncertainties. This means that the uncertainties in the models and the parameter values are considered to overwhelm the statistical uncertainty, an assumption which is reasonable in the light of the current state of the art.

Most of the parameters that must be used as input to COMPBRN are either fixed by the problem, e.g., its geometry, or are known to a satisfactory degree. On the other hand, significant uncertainties (at least to the extent that they must be represented by probability distributions) exist regarding the thermal and combustion properties of cable insulation and jacket material. The principal sources of uncertainty are the lack of information regarding the composition of these materials and of experimental evidence.

To propagate the parameter uncertainties, either Monte Carlo methods or response surface methods can be used. The purpose of the response surface is to replace the expensive computer code with a much simpler analytical expression, 
that is, to represent the output of a computer code by a simple function of its inputs. The form of the specific response surface depends on the specific application. The coefficients in the response surface are estimated by using the data from a few runs of the computer code.

To treat the modeling uncertainties, Ref. 6 proposes to use an "error" or "uncertainty" factor $E_{\tau}$, which is multiplied with the code prediction to yield the actual growth time. Because the exact value of $\mathrm{E}_{\mathrm{T}}$ is unknown for an arbitrary scenario, we work with its probability distribution. In Reference 6 , $E_{T}$ is assumed to be lognormally distributed with a 5 th percentile of 0.8 and a 95 th percentile of 4.0 , thus recognizing that COMPBRN yields generally conservative results. These percentiles are loosely based upon comparison of early simulations of cable tray fires with experimental values and, mainly, expert opinion.

The modeling uncertainty must, of course, be revised as the code is updated or as more evidence becomes available. For example, a recent modification of COMPBRN consists of the inclusion of heat losses due to radiation and convection, at a component's surface (these losses were neglected in the first version of the code), and the relaxation of the assumption that components are damaged only when they are ignited (12). Consequently, a new distribution for the uncertainty factor must be used in connection with this new version of the code.

The presence of both parameter and modeling uncertainties has an additional consequence. When experimental results become available, it is not clear how the code can be used to simulate them. Ideally, of course, we would like to know all the exact values of the parameters of the experiments, in which case the modeling approximations of the code can be tested. In practice, however, such is not the case and how exactly one should proceed is not well understood.

\section{DETECTION AND SUPPRESSION}

The issues that arise in developing the distribution of the hazard time $\mathrm{T}_{\mathrm{H}}$ of eq. (1) are, to a large extent, similar to those that we have discussed. This, of course, is not surprising, since the root causes are the same, i.e., the data are sparse and vague. Often, however, we can infer qualltative levels of a few characteristics. For example, we may be able to roughly judge the size of a fire (at detection) based upon what type of fuel (which determines the intensity) and what type of component (which determines the burning area) was involved. Similarly, the growth rate of the fire can be assessed by determining the fuel involved and the ignition source.

\section{CONCLUDING REMARKS}

We have discussed several af the issues that arise when the risk associated with fires in nuclear power plants is quantified. These issues occur naturally, when one attempts to develop a methodology, which combines deterministic models, statistical evidence, and judgment. The methodology is relatively new and has not had the benefit of extensive applications and peer reviews. As such, it is continually evolving; nevertheless, its basic elements appear to be sound and its use provides useful insights, which can be used in decision making concerning plant modifications (13). 
This work has been sponsored by the Division of Risk Analysis of the W. S. Nuclear Regulatory Commission. I thank B. Buchbinder for his continuing support. Two of my former graduate students, M. Kazarians and N. Siu, who participated in the early stages of the project and made substantial contributions, deserve special mention.

\section{REFERENCES}

1. Indian Point Probabilistic Safety Study, Consolidated Edison Company of New York, Inc, and Power Authority of the State of New York, May 1982.

2. Kaplan, S., and Garrick, B.J., "On the Quantitative Definition of Risk," Risk Analysis, $1: 11-37,1981$.

3. Apostolakis, G., "Data Analysis in Risk Assessment", Nuclear Engineering and Design, 71: 375-381, 1982.

4. Apostolakis, G., Kazarians, M., and Bley, D.C., "Methodology for Assessing the Risk from Cable Fires," Nuclear Safety, 23: 391-407, 1982

5. Kazarians, M., Siu, N.O., and Apostolakis, G., "Fire Risk Analysis for Nuclear Power Plants: Methodological Developments and Applications," Risk Analysis, 5: 33-51, 1985

6. Siu, N.O., and Apostolakis, G., "Probabilistic Models for Cable Tray Fires," Reliability Engineering, 3: 213-227, 1982.

7. Siu, N.0., "Physical Models for Compartment Fires," Reliability Engineering, 3: $229-252,1982$.

8. Siu, N. and Apostolakis, G., "Modeling the Detection and Suppression of Fires in Nuclear Power Plants," Presented at the American Nuclear Society/European Nuclear Society Topical Meeting on Probabilistic Safety Methods and Applications, San Francisco, Galifornia, February 24 - March 1, 1985.

9. Kazarians, M. and Apostolakis, G., "Modeling Rare Events: The Frequencies of Fires in Nuclear Power Plants," in: Low-Probability/High-Consequence Risk Analysis, Waller, R.A., and V.T. Covello, Editors, Plenum Press, New York, 1984.

10. Kaplan, S., "On a 'Two-Stage' Bayesian Procedure for Determining Failure Rates from Experimenta1 Data", IEEE Transactions on Power Apparatus and Systems, PAS-102: 195-202, $198 \overline{3}$.

11. de Finetti, B., Theory of Probability, Vols, 1 and 2, John Wiley \& Sons, New York, 1974.

12. Chung, G., Siu, N., and Apostolakis, G., "Improvements in Compartment Fire Modeling and Simulation of Experiments," Nuclear Technology, 69: 14-26, 1985

13. Kazarians, M., Siu, N.O., and Apostolakis, G., "Application of Nuclear Power Plant Fire Risk Analysis to Risk Management," Presented at the First International Symposium on Fire Safety Science National. Bureau of Standards, Gaithersburg, Maryland, October 9-11, 1985. 